\title{
Planar Orthogonal Polynomials As Type II Multiple Orthogonal Polynomials
}

\author{
Seung-Yeop Lee, Meng Yang
}

\begin{abstract}
We show that the planar orthogonal polynomials with $l$ logarithmic singularities in the potential are the multiple orthogonal polynomials (Hermite-Padé polynomials) of Type II with $l$ measures. We also find the ratio between the determinant of the moment matrix corresponding to the multiple orthogonal polynomials and the determinant of the moment matrix from the original planar measure.
\end{abstract}

\section{Main Result}

Let $p_{n}(z)$ be the monic polynomial of degree $n$ satisfying the orthogonality:

$$
\int_{\mathbb{C}} p_{n}(z) \overline{p_{m}(z)} \mathrm{e}^{-|z|^{2}}|W(z)|^{2} \mathrm{~d} A(z)=h_{n} \delta_{n m}, \quad n, m \geq 0,
$$

where $\mathrm{d} A$ is the Lebesgue area measure of the complex plane and $h_{n}$ is the positive norming constant. We define, for $l \geq 1$, the multi-valued function $W$ by

$$
W(z)=\prod_{j=1}^{l}\left(z-a_{j}\right)^{c_{j}}, \quad z \in \mathbb{C},
$$

where $\left\{c_{1}, \cdots, c_{l}\right\}$ are positive real numbers and $\left\{a_{1}, \cdots, a_{l}\right\}$ are distinct points in $\mathbb{C}$.

The orthogonal polynomial whose measure is supported on the plane is called planar orthogonal polynomial. Such polynomial has been of interest due to its connection to two-dimensional Coulomb gas [1. Moreover the polynomial defined above appears [2] in the quantized version of Hele-Shaw flow, a type of growth model in the two-dimensional plane. These connections to physical system, Coulomb gas and Hele-Shaw flow, motivate one to study the large degree behavior of the polynomials. We recommend the recent paper 3 for an important progress in this regard and for the related history. Still lacking, until now, is the understanding of the limiting zero distribution when the degree of the polynomial goes to infinity. Several case studies [4, 5, 6, 7, 8, 9, have shown that the zeros tend to certain one-dimensional set. In all of these cases the planar orthogonal polynomial in question turns out to be also either a classical orthogonal polynomial or a multiple orthogonal polynomial [10, 11, of which the asymptotic behavior is possible to study [13] due to rich algebraic structure such as finite term recurrence relation.

The main result of the paper is that our polynomials $\left\{p_{n}\right\}$ are multiple orthogonal polynomials of Type II. To introduce the main theorem, let us prepare several notations. To remove the unnecessary complication, we assume that $a_{j}$ 's are all nonzero and the arguments of $a_{j}$ 's are all different. Without loss of generality, we may assume:

$$
0 \leq \arg a_{1}<\cdots<\arg a_{l}<2 \pi .
$$



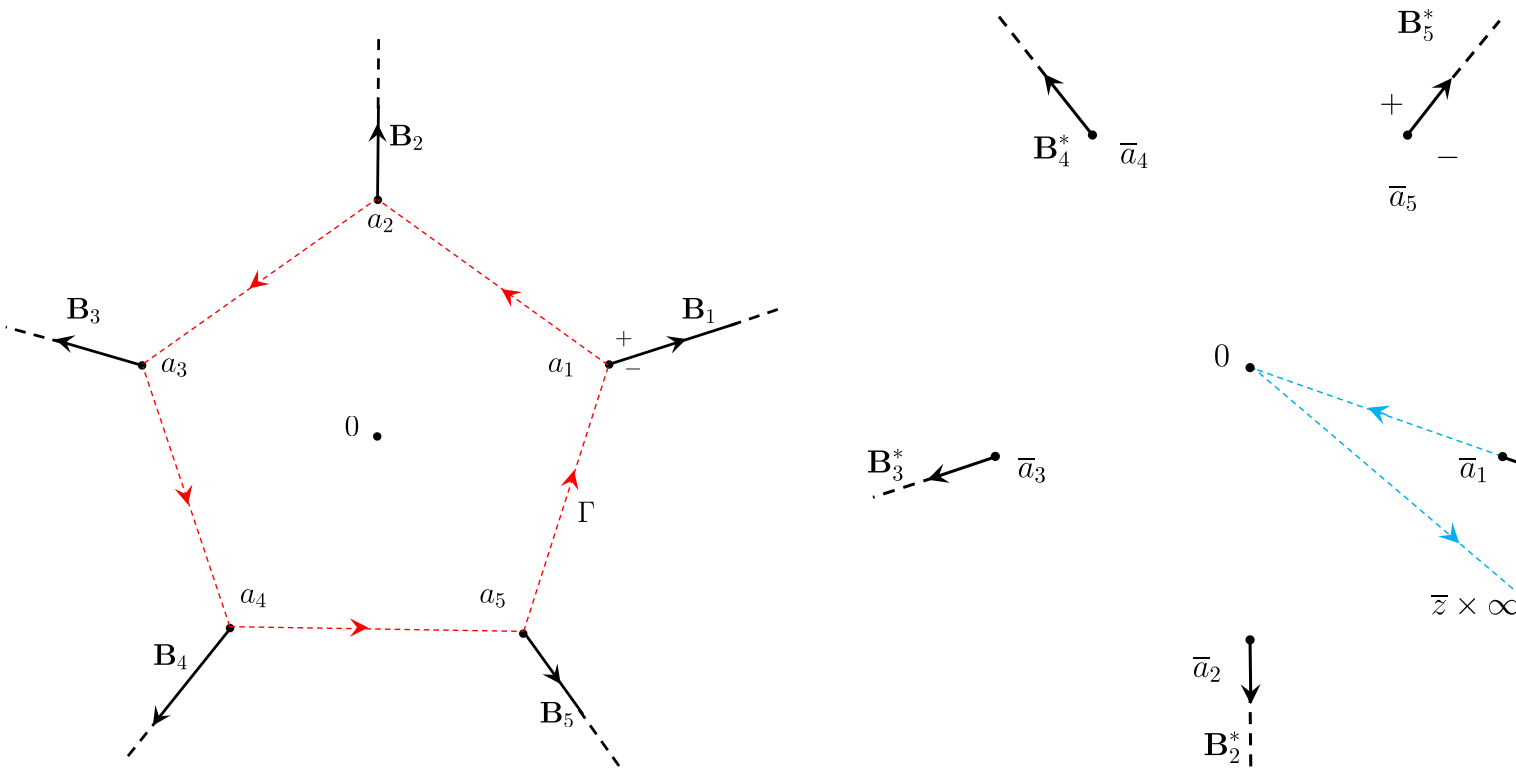

$\bar{a}_{5}$
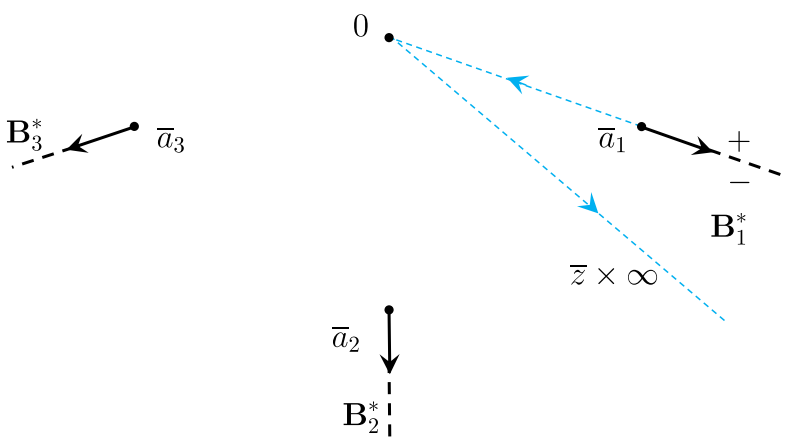

Figure 1: Contours when $l=5$. In the left are contours for $\mathbf{B}$ (black) and $\Gamma$ (dotted red); In the right are the complex conjugate image of the right, and the integration contour for $\tilde{\chi}_{\mathbf{k}}$ (dotted blue).

To determine the branch of the multi-valued function $W$, we define the union of contours,

$$
\mathbf{B}=\bigcup_{j=1}^{l} \mathbf{B}_{j}, \quad \mathbf{B}_{j}=\left\{a_{j} t: t \geq 1\right\}
$$

where the contours are directed towards the infinity. In the rest of the paper, we define $W: \mathbb{C} \backslash \mathbf{B} \rightarrow \mathbb{C}$ be an analytic branch of (2). Let $\mathbf{B}^{*}$ and $\mathbf{B}_{j}^{*}$ be the complex-conjugate images of $\mathbf{B}$ and $\mathbf{B}_{j}$. Let $\bar{W}: \mathbb{C} \backslash \mathbf{B}^{*} \rightarrow \mathbb{C}$ be defined by

$$
\bar{W}(z)=\overline{W(\bar{z})}=\prod_{j=1}^{l}\left(z-\bar{a}_{j}\right)^{c_{j}} .
$$

Let $\mathbf{k}=\left(k_{1}, \cdots, k_{l}\right)$ with non-negative integers $k_{j}$ 's. When $\arg z \notin\left\{\arg a_{1}, \cdots, \arg a_{l}\right\}$, we define

$$
\chi_{\mathbf{k}}(z)=W(z) \int_{0}^{\bar{z} \times \infty} \prod_{j=1}^{l}\left(s-\bar{a}_{j}\right)^{k_{j}} \bar{W}(s) e^{-z s} \mathrm{~d} s,
$$

where the represented integration contour is $\{\bar{z} t \mid t \geq 0\}$.

Definition 1. Let $\Gamma$ be a simple closed curve with counterclockwise orientation, that connects $\left\{a_{1}, \cdots, a_{l}\right\}$, encloses the origin, and does not intersect $\mathbf{B} \backslash\left\{a_{1}, \cdots, a_{l}\right\}$. Explicitly, we may choose $\Gamma=\overline{a_{1} a_{2}} \cup \cdots \cup$ $\overline{a_{l-1} a_{l}} \cup \overline{a_{l} a_{1}}$, by the union of $l$ line segments.

Definition 2. Let $\mathbf{n}=\left(n_{1}, \cdots, n_{l}\right)$ with non-negative integers $n_{j}$ 's. We define $p_{\mathbf{n}}(z)$ to be the monic polynomial of degree $|\mathbf{n}|=n$ satisfying the orthogonality condition:

$$
\int_{\Gamma} p_{\mathbf{n}}(z) z^{k} \chi_{\mathbf{n}-\mathbf{e}_{j}}(z) \mathrm{d} z=0, \quad 0 \leq k \leq n_{j}-1, \quad 1 \leq j \leq l .
$$


Here $\mathbf{e}_{j}$ is the unit vector with one at the $j$ th entry and zeros at all the other entries. We define $q_{\mathbf{n}}^{(i)}(z)$ to be the monic polynomial of degree $|\mathbf{n}|-1$ satisfying the orthogonality condition:

$$
\int_{\Gamma} q_{\mathbf{n}}^{(i)}(z) z^{k} \chi_{\mathbf{n}-\mathbf{e}_{j}}(z) \mathrm{d} z=0, \quad 0 \leq k \leq n_{j}-1-\delta_{i j}, \quad 1 \leq i, j \leq l .
$$

The polynomials $p_{\mathbf{n}}(z)$ and $q_{\mathbf{n}}^{(i)}(z)$ are multiple orthogonal polynomials of type II.

Multiple orthogonal polynomials are related to Hermite-Padé approximation to a system of Markov functions [12]. For type II Hermite-Padé approximation, we look for rational functions approximating Markov functions near infinity, which consists of finding a polynomial $P_{\mathbf{n}}$ of degree $|\mathbf{n}|$ and polynomials $Q_{\mathbf{n}, j}(j=1, \cdots, l)$ of degree less than $|\mathbf{n}|$ such that

$$
P_{\mathbf{n}}(z) f_{j}(z)-Q_{\mathbf{n}, j}(z)=\mathcal{O}\left(\frac{1}{z^{n_{j}+1}}\right), \quad z \rightarrow \infty, j=1, \cdots, l,
$$

where $f_{1}, \cdots, f_{l}$ are $l$ Markov functions given, in our context, by

$$
f_{j}(z)=\int_{\Gamma} \frac{\chi_{\mathbf{n}-\mathbf{e}_{j}}(s)}{z-s} \mathrm{~d} s, \quad z \notin \Gamma, j=1 \cdots, l .
$$

Then $Q_{\mathbf{n}, j}(z)$ is given by

$$
Q_{\mathbf{n}, j}(z)=\int_{\Gamma} \frac{\left(P_{\mathbf{n}}(z)-P_{\mathbf{n}}(s)\right) \chi_{\mathbf{n}-\mathbf{e}_{j}}(s)}{z-s} \mathrm{~d} s .
$$

In our context, $P_{\mathbf{n}}=p_{\mathbf{n}}$. We now state the main results:

Theorem 1. Given positive integers $n$ and $l$, we define a non-negative integer $\kappa$ and a non-negative integer $0 \leq r<l$ such that $n=\kappa l+r$. Then,

$$
p_{n}(z)=p_{\mathbf{n}}(z),
$$

where $\mathbf{n}=\mathbf{n}(n, l)=(\underbrace{\kappa+1, \cdots, \kappa+1}_{r}, \underbrace{\kappa, \cdots, \kappa}_{l-r})$.

The next theorem is an immediate consequence; see [11] for a reference.

Theorem 2. Let $n, l, \kappa, r$ and $\mathbf{n}$ be given as in Theorem 1, Let the $(l+1)$ by $(l+1)$ matrix function $Y$ be given by

$Y(z)=\left[\begin{array}{cccc}p_{\mathbf{n}}(z) & \frac{1}{2 \pi \mathrm{i}} \int_{\Gamma} \frac{p_{\mathbf{n}}(w) \chi_{\mathbf{n}-\mathbf{e}_{1}}(w)}{w-z} \mathrm{~d} w & \cdots & \frac{1}{2 \pi \mathrm{i}} \int_{\Gamma} \frac{p_{\mathbf{n}}(w) \chi_{\mathbf{n}-\mathbf{e}_{l}}(w)}{w-z} \mathrm{~d} w \\ \vdots & \vdots & \vdots & \vdots \\ \gamma_{j} q_{\mathbf{n}}^{(j)}(z) & \frac{\gamma_{j}}{2 \pi \mathrm{i}} \int_{\Gamma} \frac{q_{\mathbf{n}}^{(j)}(w) \chi_{\mathbf{n}-\mathbf{e}_{1}}(w)}{w-z} \mathrm{~d} w & \cdots & \frac{\gamma_{j}}{2 \pi \mathrm{i}} \int_{\Gamma} \frac{q_{\mathbf{n}}^{(j)}(w) \chi_{\mathbf{n}-\mathbf{e}_{l}(w)}}{w-z} \mathrm{~d} w \\ \vdots & \vdots & \vdots & \vdots\end{array}\right] \leftarrow(j+1)$ th row,

where the constant $\gamma_{j}$ in the $(j+1)$ th row is given by

$$
\gamma_{j}=-\left(\frac{1}{2 \pi \mathrm{i}} \int_{\Gamma} q_{\mathbf{n}}^{(j)}(w) w^{m} \chi_{\mathbf{n}-\mathbf{e}_{j}}(w) \mathrm{d} w\right)^{-1}, \quad m=\left\{\begin{array}{l}
\kappa \quad \text { for } 1 \leq j \leq r \\
\kappa-1 \text { for } r+1 \leq j \leq l .
\end{array}\right.
$$


Then the matrix function $Y$ is the unique solution to the Riemann-Hilbert problem given below.

$$
\left\{\begin{array}{l}
Y: \mathbb{C} \backslash \Gamma \rightarrow \mathbb{C}^{(l+1) \times(l+1)} \text { is holomorphic matrix function; } \\
Y_{+}(z)=Y_{-}(z) J(z) \text { on } \Gamma ; \\
Y(z)=\left(I+\mathcal{O}\left(\frac{1}{z}\right)\right)\left[\begin{array}{ccc}
z^{n} & \mathbf{0} & \mathbf{0} \\
\mathbf{0} & z^{-(\kappa+1)} I_{r \times r} & \mathbf{0} \\
\mathbf{0} & \mathbf{0} & z^{-\kappa} I_{(l-r) \times(l-r)}
\end{array}\right], \quad \text { as } z \rightarrow \infty .
\end{array}\right.
$$

Above, the subscript \pm in $Y_{ \pm}$represents the limiting value when approaching $\Gamma$ from the corresponding sides of the directed contour, and

$$
J(z)=\left[\begin{array}{cccc}
1 & \chi_{\mathbf{n}-\mathbf{e}_{1}}(z) & \cdots & \chi_{\mathbf{n}-\mathbf{e}_{l}}(z) \\
0 & 1 & \cdots & 0 \\
\vdots & \vdots & \ddots & \vdots \\
0 & 0 & \cdots & 1
\end{array}\right] .
$$

Remark. For $l=1$, the contour $\Gamma$ is a closed curve around the origin passing through $a_{1}$. After a little computation one can see that the jump contour $\Gamma$ can be deformed to enclose the line segment $\left[0, a_{1}\right]$, to match the one in [6].

Let us define the moments,

$$
\begin{aligned}
& \nu_{j k}^{(i)}:=\frac{1}{2 \mathrm{i}} \int_{\Gamma} z^{j+k} \chi_{\mathbf{n}-\mathbf{e}_{i}}(z) \mathrm{d} z=\frac{1}{2 \mathrm{i}} \int_{\Gamma} z^{j+k} \widetilde{\chi}_{\mathbf{n}-\mathbf{e}_{i}}(z) \mathrm{d} z, \\
& \mu_{j k}:=\frac{1}{2 \mathrm{i}} \int_{\Gamma} z^{j} \chi_{k}^{\infty}(z) \mathrm{d} z=\int_{\mathbb{C}} z^{j} \bar{z}^{k} \mathrm{e}^{-|z|^{2}}|W(z)|^{2} \mathrm{~d} A(z) .
\end{aligned}
$$

Theorem 3. Let $n, l, \kappa, r$ and $\mathbf{n}=(\underbrace{\kappa+1, \cdots, \kappa+1}_{r}, \underbrace{\kappa, \cdots, \kappa}_{l-r})$ be given as in Theorem 1 , For $\nu_{j k}^{(i)}$ and $\mu_{j k}$ given above, set the $n$ by $n$ matrices of moments $d_{n}$ and $D_{n}$ by

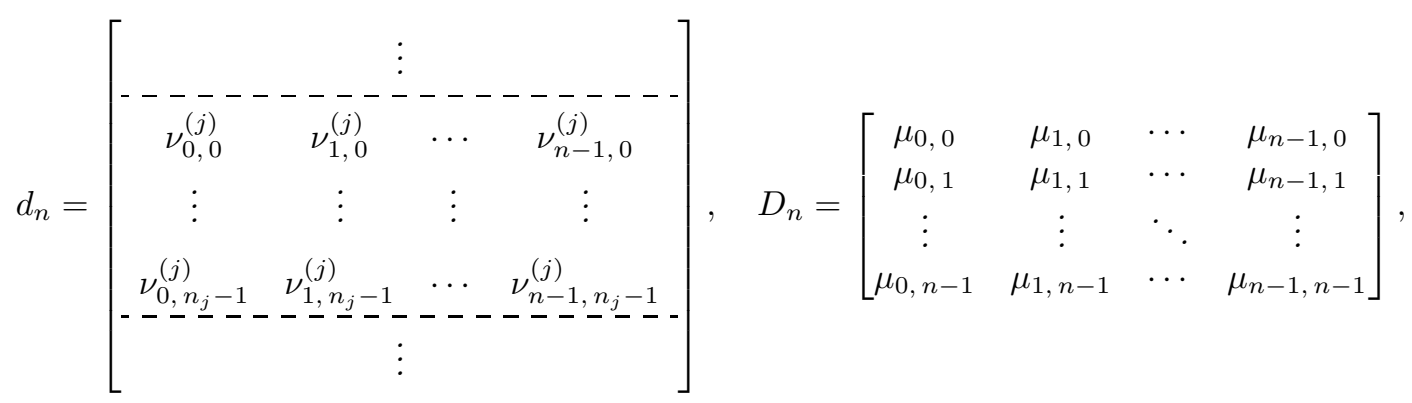

where

$$
n_{i}=\left\{\begin{array}{l}
\kappa+1 \quad \text { for } 1 \leq i \leq r \\
\kappa \quad \text { for } \quad r+1 \leq i \leq l
\end{array}\right.
$$


Then there exists a unique constant matrix $A_{n}$ such that $d_{n}=A_{n} D_{n}$. Moreover it satisfies

$$
\begin{aligned}
\operatorname{det} A_{n} & =(-1)^{n(n-1) / 2}\left(\prod_{i=1}^{l} \prod_{j=1}^{n_{i}-1}\left(c_{i}+j\right)^{j}\right) \prod_{i<j}\left(\bar{a}_{j}-\bar{a}_{i}\right)^{n_{i} n_{j}} \\
& =(-1)^{n(n-1) / 2}\left(\prod_{i=1}^{l} \prod_{j=1}^{\kappa-1}\left(c_{i}+j\right)^{j}\right)\left(\prod_{i=1}^{r}\left(c_{i}+\kappa\right)^{\kappa}\right) \prod_{1 \leq i<j \leq l}\left(\bar{a}_{j}-\bar{a}_{i}\right)^{\kappa^{2}} \\
& \times \prod_{1 \leq i<j \leq r}\left(\bar{a}_{j}-\bar{a}_{i}\right)^{2 \kappa+1} \prod_{j=r+1}^{l} \prod_{i=1}^{r}\left(\bar{a}_{j}-\bar{a}_{i}\right)^{\kappa} .
\end{aligned}
$$

Theorem 2 provides a way to study such planar orthogonal polynomials, namely, by the nonlinear steepest descent analysis of matrix Riemann-Hilbert problem, see [6, 5, 8, 9]. Theorem 3 suggests that the partition function of the corresponding Coulomb Gas system (see [14 and the reference therein) can be calculated using the tau-function from the Riemann-Hilbert problem [15. Both directions are currently in progress by the authors.

\section{Proof of Theorem 1}

\subsection{Area Integral via Contour Integral}

The following definitions will be useful.

$$
\begin{aligned}
\chi_{m}(z) & :=W(z) \int_{0}^{\bar{z}} s^{m} \bar{W}(s) \mathrm{e}^{-z s} \mathrm{~d} s, \\
\chi_{m}^{\infty}(z) & :=W(z) \int_{0}^{\bar{z} \times \infty} s^{m} \bar{W}(s) \mathrm{e}^{-z s} \mathrm{~d} s .
\end{aligned}
$$

Both are well defined if $\arg z \neq \arg a_{j}$ for all $j$. They satisfy the following lemma.

Lemma 1. Let $\mathbf{S}=\bigcup_{j=1}^{l} \mathbf{S}_{j}$ where $\mathbf{S}_{j}=\left\{a_{j} t: 0 \leq t \leq 1\right\}$. $\chi_{m}^{\infty}(z)-\chi_{m}(z)$ has continuous extension in $\mathbb{C} \backslash \mathbf{S}$ and, given $k>0$, there exists $C>0$ such that

$$
\left|z^{k}\right|\left|\chi_{m}^{\infty}(z)-\chi_{m}(z)\right| \leq C \mathrm{e}^{-(|z|-1)^{2}}
$$

for all $z$ such that $|z|>2$.

Proof. It is enough to check the continuity on $\mathbf{B}_{1} \backslash\left\{a_{1}\right\}$. The piecewise analytic functions, $W$ and $\bar{W}$, satisfy the following jump conditions,

$$
\begin{aligned}
& W_{+}(z)=\mathrm{e}^{-2 \pi \mathrm{i} c_{j}} W_{-}(z), \quad z \in \mathbf{B}_{j}, \\
& \bar{W}_{+}(z)=\mathrm{e}^{-2 \pi \mathrm{i} c_{j}} \bar{W}_{-}(z), \quad z \in \mathbf{B}_{j}^{*} .
\end{aligned}
$$

Here the subscripts \pm stand for the boundary values taken from \pm sides of $\mathbf{B}$; we assign \pm sides on each point of $\mathbf{B} \backslash\left\{a_{1}, a_{2}, \cdots, a_{l}\right\}$ and $\mathbf{B}^{*} \backslash\left\{\bar{a}_{1}, \bar{a}_{2}, \cdots, \bar{a}_{l}\right\}$ in a standard way, see Figure 1

Let $p \in \mathbf{B}_{1} \backslash\left\{a_{1}\right\}$. Note that when $z$ approaches $p$ from + side of $\mathbf{B}_{1}, \bar{z}$ approaches $\mathbf{B}_{1}^{*}$ from side. Then we get

$$
\begin{aligned}
{\left[\chi_{m}^{\infty}(p)-\chi_{m}(p)\right]_{+} } & =[W(p)]_{+} \int_{\bar{p}}^{\bar{p} \times \infty} s^{m}[\bar{W}(s)]_{-} \mathrm{e}^{-p s} \mathrm{~d} s \\
& =[W(p)]_{-} \int_{\bar{p}}^{\bar{p} \times \infty} s^{m}[\bar{W}(s)]_{+} \mathrm{e}^{-p s} \mathrm{~d} s \\
& =\left[\chi_{m}^{\infty}(p)-\chi_{m}(p)\right]_{-},
\end{aligned}
$$


where we used (12) at the second equality. This proves the continuity statement. To prove the statement about the bound, we use the elementary estimate that, given $k>0$, there exists $C>0$ such that

$$
\left|z^{k}\right||W(z)| \leq C \mathrm{e}^{|z|}
$$

for all $z \in \mathbb{C}$. Then, for some $C>0$ and $|z|>2$, we get

$$
\begin{aligned}
\left|z^{k}\left(\chi_{m}^{\infty}(z)-\chi_{m}(z)\right)\right| & =\left|z^{k} W(z) \int_{\bar{z}}^{\bar{z} \times \infty} s^{m} \bar{W}(s) \mathrm{e}^{-z s} \mathrm{~d} s\right| \\
& \leq C \mathrm{e}^{|z|} \int_{\bar{z}}^{\bar{z} \times \infty} \mathrm{e}^{|s|} \mathrm{e}^{-z s}|\mathrm{~d} s| \\
& \leq C \mathrm{e}^{|z|}\left|\int_{|\bar{z}|}^{\infty} \mathrm{e}^{x} \mathrm{e}^{-|z| x} \mathrm{~d} x\right|=C \frac{\mathrm{e}^{-|z|^{2}+2|z|}}{|z|-1} \leq \widetilde{C} \mathrm{e}^{-(|z|-1)^{2}}
\end{aligned}
$$

Proposition 1. For an arbitrary polynomial $p(z)$ we have the following identity:

$$
\int_{\mathbb{C}} p(z) \bar{z}^{m} \mathrm{e}^{-|z|^{2}}|W(z)|^{2} \mathrm{~d} A(z)=\frac{1}{2 \mathrm{i}} \int_{\Gamma} p(z) \chi_{m}^{\infty}(z) \mathrm{d} z .
$$

Proof. We apply Green's theorem to change the integral over $\mathbb{C}$ to the integral over a contour. First we observe that

$$
\bar{z}^{m}|W(z)|^{2} \mathrm{e}^{-|z|^{2}}=\frac{\partial \chi_{m}(z)}{\partial \bar{z}}, \quad z \in \mathbb{C} \backslash \mathbf{B} .
$$

Therefore, defining $D_{R}:=\{z|| z \mid<R\}$, we get

$$
\begin{aligned}
\int_{\mathbb{C}} p(z) \bar{z}^{m}|W(z)|^{2} \mathrm{e}^{-|z|^{2}} \mathrm{~d} A(z) & =\lim _{R \rightarrow \infty} \int_{D_{R}} p(z) \bar{z}^{m}|W(z)|^{2} \mathrm{e}^{-|z|^{2}} \mathrm{~d} A(z) \\
& =\lim _{R \rightarrow \infty} \int_{D_{R} \backslash \mathbf{B}} p(z) \frac{\partial \chi_{m}(z)}{\partial \bar{z}} \mathrm{~d} A(z) \\
& =\lim _{R \rightarrow \infty} \frac{1}{2 \mathrm{i}}\left(\int_{\partial D_{R}} p(z) \chi_{m}(z) \mathrm{d} z+\sum_{j=1}^{m} \int_{\mathbf{B}_{j} \cap D_{R}} p(z)\left[\chi_{m}(z)\right]_{-}^{+} \mathrm{d} z\right),
\end{aligned}
$$

where we use Green's theorem at the last equality.

Since $\chi_{m}^{(\infty)}(z)$ is analytic in $\mathbb{C} \backslash(\mathbf{S} \cup \mathbf{B})$, by deformation of contour we get the identity

$$
\int_{\Gamma} p(z) \chi_{m}^{\infty}(z) \mathrm{d} z=\int_{\partial D_{R}} p(z) \chi_{m}^{\infty}(z) \mathrm{d} z+\sum_{j=1}^{m} \int_{\mathbf{B}_{j} \cap D_{R}} p(z)\left[\chi_{m}(z)\right]_{-}^{+} \mathrm{d} z
$$

Using this identity, the right hand side of (17) becomes

$$
\lim _{R \rightarrow \infty} \frac{1}{2 \mathrm{i}} \int_{\partial D_{R}} p(z)\left(\chi_{m}(z)-\chi_{m}^{\infty}(z)\right) \mathrm{d} z+\frac{1}{2 \mathrm{i}} \int_{\Gamma} p(z) \chi_{m}^{\infty}(z) \mathrm{d} z=\frac{1}{2 \mathrm{i}} \int_{\Gamma} p(z) \chi_{m}^{\infty}(z) \mathrm{d} z,
$$

where the last equality holds because of (11) in Lemma 1, This proves Proposition 1 . 


\subsection{Several Lemmas}

Definition 3. All the vectors in this paper have only non-negative entries. For two vectors, $\mathbf{k}$ and $\mathbf{s}$, we say $\mathbf{k} \geq \mathbf{s}$ if $\mathbf{k}-\mathbf{s}$ has only non-negative entries. If, in addition, $\mathbf{k} \neq \mathbf{s}$ then we say $\mathbf{k}>\mathbf{s}$. The $j$ th entry of $\mathbf{k}$ is denoted by $[\mathbf{k}]_{j}$. We define the length of a vector by $|\mathbf{k}|=[\mathbf{k}]_{1}+\cdots+[\mathbf{k}]_{l}$.

Lemma 2. For any $n \geq 1$ we have

$$
\operatorname{span}\left\{\chi_{j}^{\infty}: 0 \leq j<n\right\}=\operatorname{span}\left\{\chi_{\mathbf{k}}:|\mathbf{k}| \leq n\right\} .
$$

Proof. For $n=0$, the lemma holds because $\chi_{0}^{\infty}(z)=\chi_{\mathbf{0}}(z)$. Assume that the lemma holds for $n=n_{0}$. If $|\mathbf{k}|=n_{0}+1$ we get

$$
\begin{aligned}
\chi_{\mathbf{k}}(z)-\chi_{n_{0}+1}^{\infty}(z) & =W(z) \int_{0}^{\bar{z} \times \infty} \prod_{j=1}^{l}\left(s-\bar{a}_{j}\right)^{k_{j}} \bar{W}(s) \mathrm{e}^{-z s} \mathrm{~d} s-W(z) \int_{0}^{\bar{z} \times \infty} s^{n_{0}+1} \bar{W}(s) \mathrm{e}^{-z s} \mathrm{~d} s \\
& =W(z) \int_{0}^{\bar{z} \times \infty}\left\{\text { polynomial in s of degree } \leq n_{0}\right\} \times \bar{W}(s) \mathrm{e}^{-z s} \mathrm{~d} s .
\end{aligned}
$$

Since the last term belongs to both spans in (20) for $n=n_{0}, \chi_{\mathbf{k}}$ belongs to the left span in (20) with $n=n_{0}+1$ and $\chi_{n_{0}+1}^{\infty}$ belongs to the right span in (20) with $n=n_{0}+1$.

To prove $p_{n}=p_{\mathbf{n}}$, one may try to show that

$$
\operatorname{span}\left\{\chi_{j}^{\infty}(z) \mid 0 \leq j<n\right\}=\operatorname{span}\left\{z^{k} \chi_{\mathbf{n}-\mathbf{e}_{j}} \mid 0 \leq k<[\mathbf{n}]_{j}, 1 \leq j \leq l\right\} .
$$

In fact, it is enough to show that the above equality up to functions $\psi$ that satisfies $\langle p, \psi\rangle=0$ for all polynomial $p$. For example, we have $\langle p, \psi\rangle=0$ for

$$
\psi(z)=W(z) \int_{0}^{\bar{a}_{1}} \prod_{j=1}^{l}\left(s-\bar{a}_{j}\right)^{k_{j}} \bar{W}(s) \mathrm{e}^{-z s} \mathrm{~d} s .
$$

Since $\psi$ is analytic in $\mathbb{C} \backslash \mathbf{B}$ and, therefore, the integration contour in $\int_{\Gamma} p(z) \psi(z) d z$ is contractible to a point. This allows us to consider, instead of $\chi_{\mathbf{k}}$ in (22),

$$
\widetilde{\chi}_{\mathbf{k}}:=\chi_{\mathbf{k}}-W(z) \int_{0}^{\bar{a}_{1}} \prod_{j=1}^{l}\left(s-\bar{a}_{j}\right)^{k_{j}} \bar{W}(s) \mathrm{e}^{-z s} \mathrm{~d} s .
$$

As a result, using Lemma 2, the proof of Theorem 1 is reduced to proving the following Proposition.

Proposition 2. For any $n \geq 1$ and $l \geq 1$ let $\mathbf{n}$ be given as in Theorem 1 , Then the following holds.

$$
\operatorname{span}\left\{\widetilde{\chi}_{\mathbf{k}}(z):|\mathbf{k}|<n\right\}=\operatorname{span}\left\{z^{k} \widetilde{\chi}_{\mathbf{n}-\mathbf{e}_{j}}(z) \mid 0 \leq k<[\mathbf{n}]_{j}, 1 \leq j \leq l\right\} .
$$

The proof of this proposition will be in the next subsection. The following Lemma is why it is useful to use $\widetilde{\chi}_{\mathbf{k}}$ instead of $\chi_{\mathbf{k}}$.

Lemma 3.

$$
z \widetilde{\chi}_{\mathbf{k}}(z)=\sum_{j=1}^{l}\left(c_{j}+k_{j}\right) \widetilde{\chi}_{\mathbf{k}-\mathbf{e}_{j}}(z) .
$$


Proof. Taking the integral of the total derivative as following, we have

$$
\begin{aligned}
0 & =W(z) \int_{\bar{a}_{1}}^{\bar{z} \times \infty} \partial_{s}\left[\prod_{j=1}^{l}\left(s-\bar{a}_{j}\right)^{c_{j}+k_{j}} \mathrm{e}^{-z s}\right] \mathrm{d} s \\
& =W(z) \int_{\bar{a}_{1}}^{\bar{z} \times \infty}\left(\sum_{j=1}^{l} \frac{c_{j}+k_{j}}{s-\bar{a}_{j}}-z\right) \prod_{j=1}^{l}\left(s-\bar{a}_{j}\right)^{c_{j}+k_{j}} \mathrm{e}^{-z s} \mathrm{~d} s \\
& =\sum_{j=1}^{l}\left(c_{j}+k_{j}\right) \widetilde{\chi}_{\mathbf{k}-e_{j}}(z)-z \widetilde{\chi}_{\mathbf{k}}(z) .
\end{aligned}
$$

Corollary 1. Let $\mathbf{k}=\left(k_{1}, k_{2}, \cdots, k_{l}\right)$ and $s \leq \min \left\{k_{j}\right\}_{j=1}^{l}$ be a positive integer. Then $z^{s} \widetilde{\chi}_{\mathbf{k}}(z)$ can be represented as a linear combination of $\left\{\widetilde{\chi}_{\mathbf{k}-\mathbf{s}}(z)|| \mathbf{s} \mid=s\right\}$. Furthermore, the coefficient of $\widetilde{\chi}_{\mathbf{k}-s \mathbf{e}_{m}}(z)$ is nonzero for all $1 \leq m \leq l$.

Proof. From Lemma 3, the corollary is true when $s=1$. Assume, for some $1 \leq s<\min \left\{k_{j}\right\}_{j=1}^{l}$, that $z^{s} \widetilde{\chi}_{\mathbf{k}}(z)$ is a linear combination of $\widetilde{\chi}_{\mathbf{k}-\mathbf{s}}(z)$ for $|\mathbf{s}|=s$ and the coefficient of $\left\{\widetilde{\chi}_{\mathbf{k}-s \mathbf{e}_{m}}(z)\right\}_{m=1}^{l}$ are all non-vanishing.

Then $z^{s+1} \widetilde{\chi}_{\mathbf{k}}(z)$ is a linear combination of $z \widetilde{\chi}_{\mathbf{k}-\mathbf{s}}(z)$ and, therefore, of $\widetilde{\chi}_{\mathbf{k}-\mathbf{s}-\mathbf{e}_{m}}(z)$ with $|\mathbf{s}|=s$ and $1 \leq m \leq l$. Since the term $\widetilde{\chi}_{\mathbf{k}-(s+1) \mathbf{e}_{m}}(z)$ comes only from $z \widetilde{\chi}_{\mathbf{k}-s \mathbf{e}_{m}}(z)$ and since the coefficient of $\widetilde{\chi}_{\mathbf{k}-s \mathbf{e}_{m}}(z)$ is non-zero, the coefficient of $\widetilde{\chi}_{\mathbf{k}-(s+1) \mathbf{e}_{m}}(z)$ is non-zero. Note that all the coefficients in the right hand side of (25) are non-zero. By induction, this ends the proof.

Lemma 4. For $n \neq m$, we have

$$
\tilde{\chi}_{\mathbf{k}+\mathbf{e}_{n}}(z)-\tilde{\chi}_{\mathbf{k}+\mathbf{e}_{m}}(z)+\left(\bar{a}_{n}-\bar{a}_{m}\right) \tilde{\chi}_{\mathbf{k}}(z)=0 .
$$

Proof. Since

$$
\left(s-\bar{a}_{n}\right)-\left(s-\bar{a}_{m}\right)+\left(\bar{a}_{n}-\bar{a}_{m}\right)=0,
$$

we obtain,

$$
0=W(z) \int_{\bar{a}_{1}}^{\bar{z} \times \infty}\left[\left(s-\bar{a}_{n}\right)-\left(s-\bar{a}_{m}\right)+\left(\bar{a}_{n}-\bar{a}_{m}\right)\right] \prod_{j=1}^{l}\left(s-\bar{a}_{j}\right)^{c_{j}+k_{j}} \mathrm{e}^{-z s} \mathrm{~d} s .
$$

By the definition of $\widetilde{\chi}_{\mathbf{k}}(z),(26)$ holds.

\subsection{Proof of Proposition 2}

By Corollary 1, we get $\supset$. To prove $\subset$, we note that any vector $\mathbf{k}$ can be uniquely represented as

$$
\mathbf{k}=\mathbf{n}+\mathbf{m}-\mathbf{s},
$$

where $[\mathbf{m}]_{j}[\mathbf{s}]_{j}=0$, i.e., $\mathbf{m}$ and $\mathbf{s}$ cannot be both non-vanishing in any of the entries. It is then enough to show the following claim.

Claim: For all $\mathbf{s} \leq \mathbf{n}$ and $\mathbf{m}$ satisfying $|\mathbf{n}+\mathbf{m}-\mathbf{s}|<n$,

$$
\tilde{\chi}_{\mathbf{n}+\mathbf{m}-\mathbf{s}} \in \operatorname{span}\left\{z^{k} \widetilde{\chi}_{\mathbf{n}-\mathbf{e}_{j}}(z) \mid 0 \leq k<[\mathbf{n}]_{j}, 1 \leq j \leq l\right\} .
$$


We prove this claim in two steps.

Step 1: For all $\mathbf{0}<\mathbf{s} \leq \mathbf{n}, \widetilde{\chi}_{\mathbf{n}-\mathbf{s}} \in \operatorname{span}\left\{z^{k} \widetilde{\chi}_{\mathbf{n}-\mathbf{e}_{j}}(z) \mid 0 \leq k<[\mathbf{n}]_{j}, 1 \leq j \leq l\right\}$. If $|\mathbf{s}|=1$ then the inclusion is immediate. Let the inclusion holds for $|\mathbf{s}| \leq m-1$ for some $m<n$. (If $m \geq n$ then the proof is done.) Below we claim that the inclusion holds for $|\mathbf{s}|=m$, which proves Step 1 by induction.

1. If $\mathbf{s}$ has more than one non-zero entries, i.e., $[\mathbf{s}]_{i} \neq 0$ and $[\mathbf{s}]_{j} \neq 0$,

$$
\tilde{\chi}_{\mathbf{n}-\mathbf{s}}(z)=\frac{1}{\bar{a}_{i}-\bar{a}_{j}}\left(\widetilde{\chi}_{\mathbf{n}-\mathbf{s}+\mathbf{e}_{j}}(z)-\tilde{\chi}_{\mathbf{n}-\mathbf{s}+\mathbf{e}_{i}}(z)\right) .
$$

The left hand side belongs to the span in Claim since the right hand side does by assumption.

2. If $\mathbf{s}$ has exactly one non-zero entry, i.e., $\mathbf{s}=m \mathbf{e}_{j}$ for some $j$. From $\mathbf{s}<\mathbf{n}$ we have $m \leq[\mathbf{n}]_{j}$. Since $z^{m-1} \widetilde{\chi}_{\mathbf{n}-\mathbf{e}_{j}}(z)$ is a linear combination of $\left\{\widetilde{\chi}_{\mathbf{n}-\widetilde{\mathbf{s}}}:|\widetilde{\mathbf{s}}|=m\right\}$ where the term $\widetilde{\chi}_{\mathbf{n}-m \mathbf{e}_{j}}$ appears with non-zero coefficient (see Corollary 1), and since all the other terms in the linear combination belongs to the span by item 10, $\tilde{\chi}_{\mathbf{n}-m \mathbf{e}_{j}}$ also belongs to the span in Claim.

Step 2: Step 1 showed Claim for $|\mathbf{m}|=0$. Assume that Claim is true when $|\mathbf{m}| \leq k-1$. We will show that Claim holds when $|\mathbf{m}| \leq k$, i.e. $\widetilde{\chi}_{\mathbf{n}+\mathbf{m}-\mathbf{s}}$ belongs to the span in Claim for $|\mathbf{m}|=k$. Let $\mathbf{m}$ satisfy $|\mathbf{m}|=k \geq 1$. There exists $j$ such that $[\mathbf{m}]_{j}>0$. Then $\widetilde{\chi}_{\mathbf{n}+\left(\mathbf{m}-\mathbf{e}_{j}\right)-\mathbf{s}}$ belongs to the span in the claim by the assumption. Since $\left|\mathbf{n}+\left(\mathbf{m}-\mathbf{e}_{j}\right)-\mathbf{s}\right|<n-1$ we have $|\mathbf{s}|>0$ and there exists $i \neq j$ such that $[\mathbf{s}]_{i}>0$. Then $\widetilde{\chi}_{\mathbf{n}+\left(\mathbf{m}-\mathbf{e}_{j}\right)-\left(\mathbf{s}-\mathbf{e}_{i}\right)}$ also belongs to the span by the assumption. Since, by Lemma 4 we have

$$
\tilde{\chi}_{\mathbf{n}+\mathbf{m}-\mathbf{s}}=\tilde{\chi}_{\mathbf{n}+\left(\mathbf{m}-\mathbf{e}_{j}\right)-\left(\mathbf{s}-\mathbf{e}_{i}\right)}+\left(\bar{a}_{i}-\bar{a}_{j}\right) \widetilde{\chi}_{\mathbf{n}+\left(\mathbf{m}-\mathbf{e}_{j}\right)-\mathbf{s}},
$$

the left hand side belongs to the span. This ends the proof of Proposition 2 and Theorem 1.

\section{Proof of Theorem 3}

Since $\operatorname{det} D_{n}=\prod_{j=0}^{n-1} h_{j}>0$ where $h_{j}$ is defined in (1), $D_{n}$ is an invertible matrix and this proves the existence and the uniqueness of $A_{n}$. In the remainder of the proof, we will construct $A_{n}$ using induction. Let us consider the $j$ th column of $d_{n}$,

$$
\left[\begin{array}{c}
\nu_{j, 0}^{(1)} \\
\nu_{j, 1}^{(1)} \\
\vdots \\
\nu_{j, n_{1}-1}^{(1)} \\
\vdots \\
\nu_{j, 0}^{(l)} \\
\nu_{j, 1}^{(l)} \\
\vdots \\
\nu_{j, n_{l}-1}^{(l)}
\end{array}\right]=\frac{1}{2 \mathrm{i}} \int_{\Gamma} z^{j} V_{\mathbf{n}}(z) \mathrm{d} z, \quad \text { where } V_{\mathbf{n}}=V_{\mathbf{n}}(z)=\left[\begin{array}{c}
\chi_{\mathbf{n}-\mathbf{e}_{1}} \\
z \chi_{\mathbf{n}-\mathbf{e}_{1}} \\
\vdots \\
z^{n_{1}-1} \chi_{\mathbf{n}-\mathbf{e}_{1}} \\
\vdots \\
\chi_{\mathbf{n}-\mathbf{e}_{l}} \\
z \chi_{\mathbf{n}-\mathbf{e}_{l}} \\
\vdots \\
z^{n_{l}-1} \chi_{\mathbf{n}-\mathbf{e}_{l}}
\end{array}\right] .
$$

We will find a constant $(n+1)$ by $(n+1)$ matrix $B_{n}$ such that, for all $z$,

$$
B_{n} V_{\mathbf{n}+\mathbf{e}_{r+1}}(z)=\left[\begin{array}{c}
\chi_{\mathbf{n}}(z) \\
\hdashline V_{\mathbf{n}}(z)
\end{array}\right] \text {. }
$$


This means that

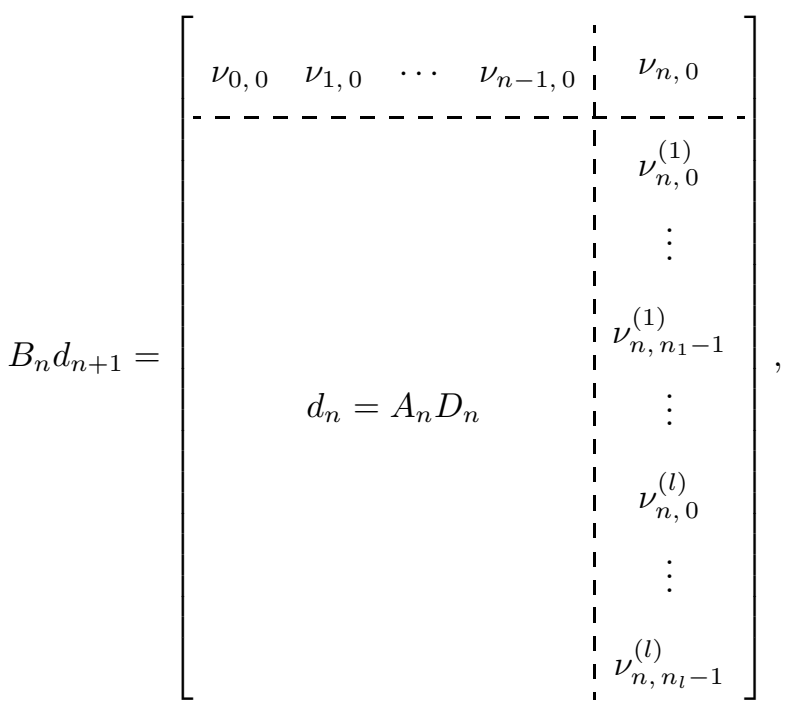

where $\nu_{j, 0}$ is given by $\nu_{j, 0}=\frac{1}{2 \mathrm{i}} \int_{\Gamma} z^{j} \chi_{\mathbf{n}}(z) \mathrm{d} z$. The matrix $B_{n}$ can be obtained by three successive linear transformations on $V_{\mathbf{n}+\mathbf{e}_{r+1}}$ that we describe below.

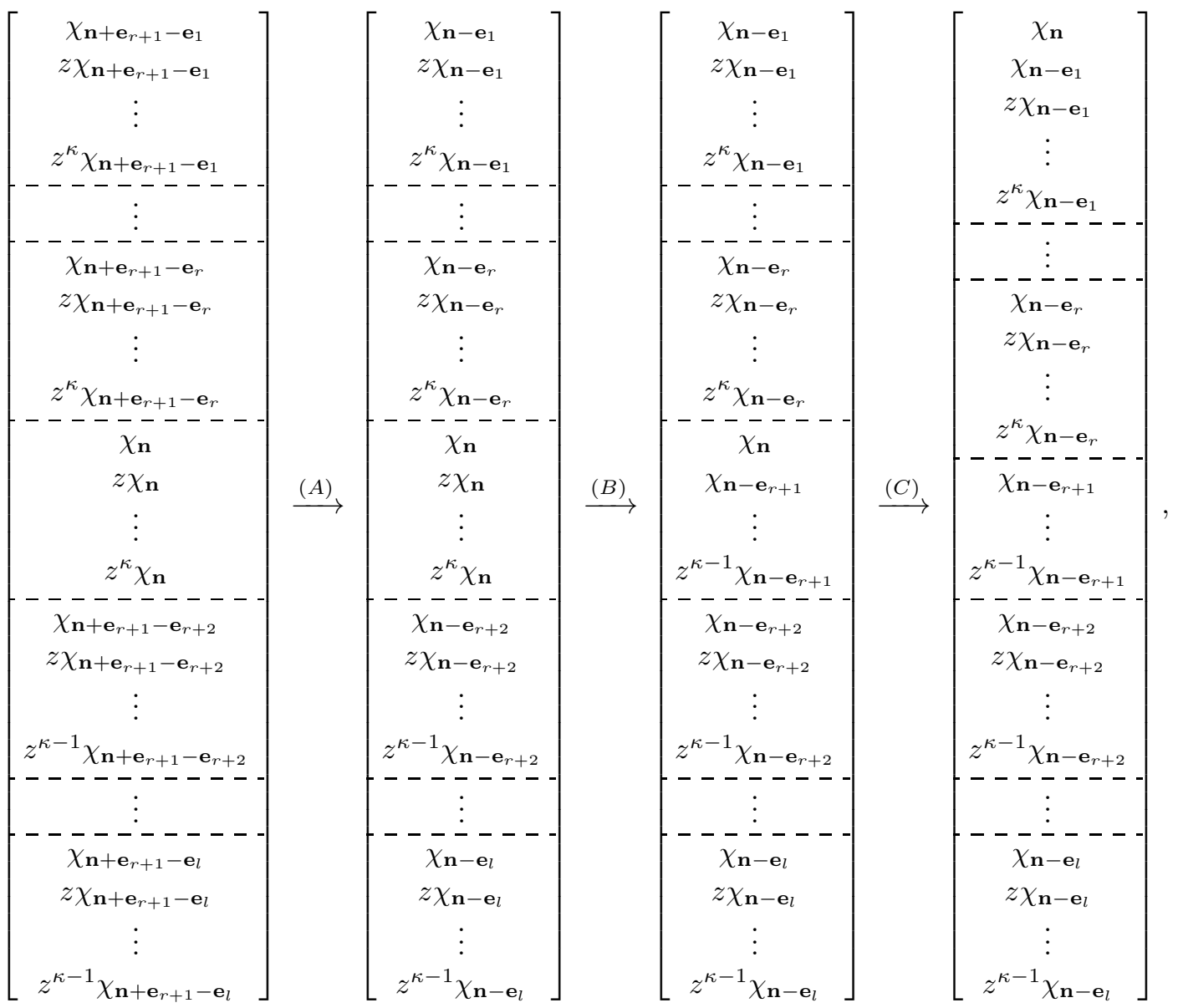


Above, each arrow means the linear transformation given by

$$
\begin{aligned}
& B_{n}^{(1)} \text { LHS of }(\mathrm{A})=\text { RHS of }(\mathrm{A}), \\
& B_{n}^{(2)} \text { LHS of (B) = RHS of (B), } \\
& B_{n}^{(3)} \operatorname{LHS} \text { of }(\mathrm{C}) \quad=\quad \text { RHS of }(\mathrm{C}) \text {, }
\end{aligned}
$$

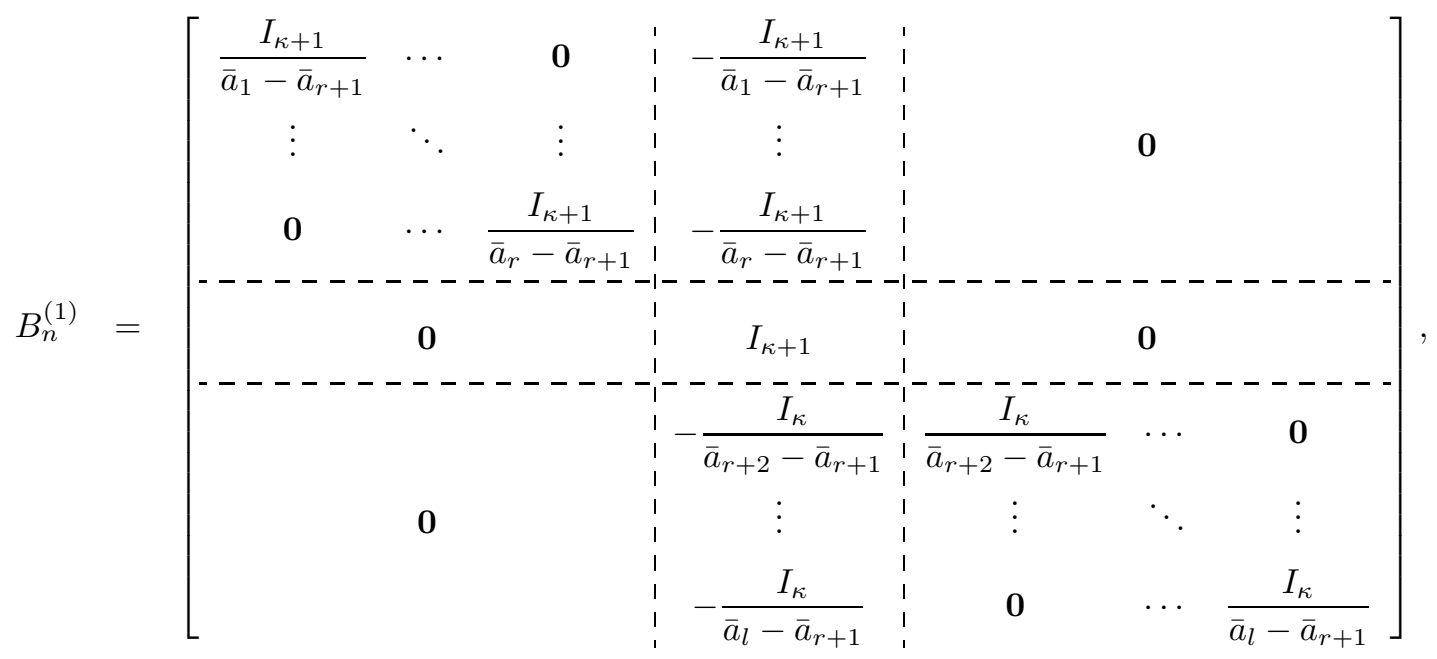

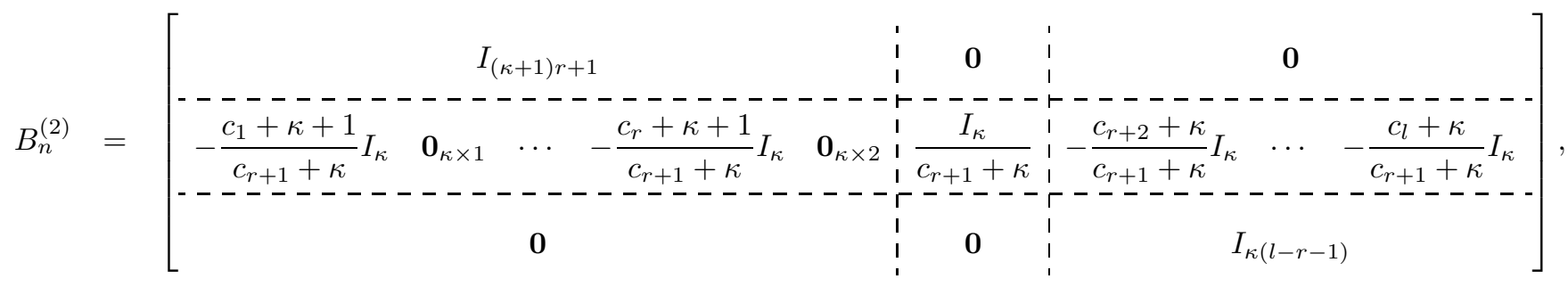

$$
\begin{aligned}
& B_{n}^{(3)}=\left[\begin{array}{c:c:c}
\mathbf{0} & 1 & \mathbf{0} \\
\hdashline I_{(\kappa+1) r} & \mathbf{0}_{(\kappa+1) \times 1} & \mathbf{0} \\
\hdashline \mathbf{0} & \mathbf{0} & I_{\kappa(l-r)}
\end{array}\right]
\end{aligned}
$$

where $I_{m}$ is the $m$ by $m$ identity matrix and $\mathbf{0}_{j \times k}$ is the zero matrix of size $j$ by $k$. We used Lemma 4 in the transformation $(A)$ and Lemma 3 in $(B)$. This gives $B_{n}=B_{n}^{(3)} B_{n}^{(2)} B_{n}^{(1)}$.

Using $d_{n}=A_{n} D_{n}$ we obtain that

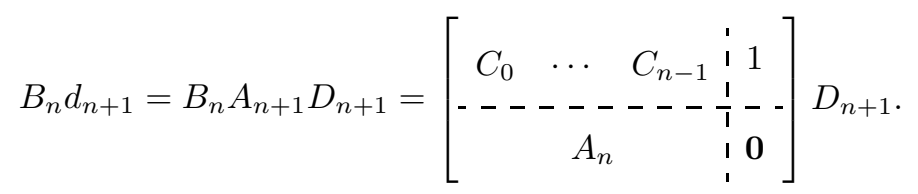

The identity at the first row is obtained by

$$
\nu_{j, 0}=\frac{1}{2 \mathrm{i}} \int_{\Gamma} z^{j} \chi_{\mathbf{n}}(z) \mathrm{d} z=\frac{1}{2 \mathrm{i}} \int_{\Gamma} z^{j} \sum_{k=0}^{n} C_{k} \chi_{k}^{\infty}(z) \mathrm{d} z=\sum_{k=0}^{n} C_{k} \mu_{j k},
$$


where $C_{k}$ is given by $\prod_{i=1}^{l}\left(z-\bar{a}_{i}\right)^{n_{j}}=\sum_{k=0}^{n} C_{k} z^{k}$. We also used that the upper $n$ by $n$ diagonal submatrix of $D_{n+1}$ is $D_{n}$.

Taking the determinant of (27) and using $B_{n}=B_{n}^{(3)} B_{n}^{(2)} B_{n}^{(1)}$, we get

$$
\begin{aligned}
& \operatorname{det} A_{n+1}=(-1)^{(n+2)}\left(\operatorname{det} B_{n}^{(1)} \operatorname{det} B_{n}^{(2)} \operatorname{det} B_{n}^{(3)}\right)^{-1} \operatorname{det} A_{n} \\
= & (-1)^{(n+2)+\sum_{i \leq r} n_{i}}\left(\prod_{i<r+1}\left(\bar{a}_{i}-\bar{a}_{r+1}\right)^{n_{i}}\right)\left(\prod_{j>r+1}\left(\bar{a}_{r+1}-\bar{a}_{j}\right)^{n_{j}}\right)\left(c_{r+1}+\kappa\right)^{\kappa} \operatorname{det} A_{n} .
\end{aligned}
$$

Now we prove (9) by induction. When $\mathbf{n}=(1,0 \cdots, 0)$ (i.e. $\kappa=0$ and $r=1$ ), by the definition of $\nu_{j k}^{(i)}$ and $\mu_{j k}$, we observe $\nu_{0,0}^{(1)}=\mu_{0,0}$. This proves $d_{1}=D_{1}$ with $\operatorname{det} A_{1}=1$. If (9) holds up to $n \leq N$ then (9) holds for $n=N+1$ by (28). Remember that if $\mathbf{n}(N, l)=\left(n_{1}, \cdots, n_{l}\right)$ and $N=\kappa l+r$ then $\mathbf{n}(N+1, l)=\left(n_{1}, \cdots, n_{r+1}+1, \cdots, n_{l}\right)$, increasing only the $(r+1)$ th entry by one. This ends the proof of Theorem 3 ,

\section{References}

[1] Y. Ameur, H. Hedenmalm, N. Makarov, Fluctuations of Eigenvalues of Random Normal Matrices, Duke Math. J., Vol.159, No.1:31-81, (2011).

[2] R. Teodorescu, E. Bettelheim, O. Agam, A. Zabrodin, P. Wiegmann, Normal randommatrix ensemble as a growth problem, Nuclear Phys. B 704, No.3, 407C444, (2005).

[3] H. Hedenmalm, A. Wennman, Planar orthogonal polynomials and boundary universality in the random normal matrix model, (arXiv:1710.06493 $\mathrm{v} 4),(2017)$.

[4] F. Balogh, T. Grava, D. Merzi, Orthogonal polynomials for a class of measures with discrete rotational symmetries in the complex plane, Constr. Approx. 46: 109. (2017)

[5] P.M. Bleher, A. Kuijlaars, Orthogonal polynomials in the normal matrix model with a cubic potential, Adv. Math. 230, 1272-1321, (2012).

[6] F. Balogh, M. Bertola, S.-Y. Lee, and K.T.-R. Mclaughlin. Strong asymptotics of the orthogonal polynomials with respect to a measure supported on the plane, Commun. Pur. Appl. Math., 68(1):112-172, (2015).

[7] A. Kuijlaars, G.L.F. Silva, S-curves in polynomial external fields, J. Approx. Theory 191, 1-37, (2015)

[8] A. Kuijlaars, A. Lopez, The normal matrix model with a monomial potential, a vector equilibrium problem, and multiple orthogonal polynomials on a star, Nonlinearity 28, 347-406, (2015).

[9] A. Kuijlaars, A. Tovbis, The supercritical regime in the normal matrix model with cubic potential, Adv. Math. 283, 530-587, (2015).

[10] A.M. Finkelshtein, W.V. Assche, What is ... a multiple orthogonal polynomial?, Notices of the AMS 63, no. 9, 1029-1031, (2016).

[11] A. Kuijlaars, Multiple orthogonal polynomials in random matrix theory, Proceedings of the International Congress of Mathematicians, Volume III (R. Bhatia, ed.) Hyderabad, India, 1417-1432, (2010).

[12] W.V. Assche, J.S. Geronimo, A. Kuijlaars, Riemann-Hilbert problems for multiple orthogonal polynomials, special functions 2000: Current Perspective and Future Directions. NATO Science Series (Series II: Mathematics, Physics and Chemistry), vol 30. 23-59, Springer, Dordrecht.(2001) 
[13] E.A. Rakhmanov, Zero distribution for Angelesco Hermite-Padé polynomials, arXiv:1712.07055v1, (2017).

[14] S.Y. Lee, M. Yang, Discontinuity in the asymptotic behavior of planar orthogonal polynomials under a perturbation of the gaussian weight, Commun. Math. Phys. vol 355: 303-338, (2017)

[15] M. Bertola, O. Marchal, The partition function of the two-matrix model as an isomonodromic $\tau$ function, J. Math. Phys. vol 50, 013529, (2009) 